\title{
AN OPTIMIZATION SCHEME FOR LOCATING POWER SYSTEM EQUILIBRIA RANKED BY A SCALAR LYAPUNOV FUNCTION
}

\author{
Christopher L. DeMarco \\ Department of Electrical and Computer Engineering \\ University of Wisconsin-Madison \\ 1415 Johnson Drive \\ Madison, WI 53706 USA
}

\begin{abstract}
Power system stability studies for swing dynamic models have long recognized the role played by unstable equilibria (u.e.p.'s) in determining the region of attraction for stable operating points. Recent works concerned with voltage instability and collapse phenomena have similarly identified the important role played by u.e.p.'s in predicting dynamic behavior in an extended class of system models. Many of these studies have required classification of unstable equilibria in terms of "distance" from the stable equilibrium of interest, with a system Lyapunov function used as the distance measure. This paper will propose a constrained optimization algorithm to implement a geometric approach to ranking u.e.p.'s. The formulation will seek to minimize a Euclidean norm of the vector field along a constraint manifold defined by a constant contour of the Lyapunov function. When the norm is driven to zero on the manifold, one has located an equilibrium point having the given value of energy. A scheme for modifying the constraint contour is proposed, and a limit on the number of local minima in a neighborhood of the operating point is demonstrated.
\end{abstract}

\section{INTRODUCTION AND BACKGROUND}

The unstable equilibrium points of a dynamical system play an important role in characterizing the qualitative behavior of its global dynamics. The utility of identifying the location and type of equilibria in electric power system stability studies has been recognized in the literature for many years; see, for example, [1] through [5]. The role of unstable equilibria is particularly clear in system models that possess a well defined Lyapunov function. As shown in [6], for many such power system models, the corresponding Lyapunov function has zero gradient at a point $\mathbf{x}$ if and only if that point is an equilibrium for the differential equations defining the system model. Transient stability studies of system models in which swing dynamics dominate have shown the importance of identifying unstable equilibria "close" to system trajectories resulting from a fault (the "controlling u.e.p." concept). More recently, work in [7] has shown that the the "closest" u.e.p., a concept largely discarded in transient stability studies, may have considerable relevance in studying voltage stibility, where the system's loss of stability is not driven by any large initiating fault. In this paper, we will examine a new approach to locating the "closest" u.e.p.; moreover, this approach will allow identification of up to $2 n$ equilibria, ranked according to the value of a system Lyapunov function evaluated at these points.
Several definitions for the "closest" u.e.p. have been examined in [5]. Here we wish to adopt a definition having a geometric basis. We will restrict our attention to systems having a well defined Lyapunov function which is locally positive definite about a known stable operating point. Positive definiteness will guarantee that in a neighborhood of the operating point, constant contours of the Lyapunov function will define a closed surface, and that increasing the value of the constant will yield a nested family of contours. The closest u.e.p. will be that unstable equilibrium first encountered by expanding these surfaces. The reader may question if these surfaces must always intersect another equilibrium as the constant is increased. Assuming the gradient of the Lyapunov function remains non-zero at points away from equilibrium (as will be demonstrated for our system models below), it can be shown that these contours must eventually intersect this closest u.e.p.

\section{REVIEW OF QUASI-GRADIENT DYNAMICAL SYSTEM STRUCTURE}

The results described in [6] suggested consideration of systems of the form

$$
\dot{\mathbf{x}}=-\mathrm{A} \nabla \mathrm{V}\left(\mathbf{x}, \mathbf{x}^{0}\right)
$$

where:

$$
V: \mathbf{R}^{\mathrm{n}} \times \mathbf{R}^{\mathrm{n}} \rightarrow \mathbf{R} \text { is continuously differentiable, }
$$$$
\mathbf{A} \in \mathbf{R}^{\mathrm{nxn}}, \operatorname{det}(\mathbf{A}) \neq 0 \text {, }
$$$$
\mathrm{V}\left(\mathbf{x}, \mathbf{x}^{0}\right)=0 \text { at } \mathbf{x}=\mathbf{x}^{0},
$$

and

$$
\nabla V\left(\mathbf{x}, \mathbf{x}^{0}\right)=\mathbf{0} \text { at } \mathbf{x}=\mathbf{x}^{0}
$$

Discussion in [6] showed that a large number of power system models in the literature could be cast in this form, and we will assume this structure for the analysis in the present paper. Here the function $\mathrm{V}\left(\mathbf{x}, \mathbf{x}^{0}\right)$ will serve as the system Lyapunov function; as noted in the introduction, equilibria of (1) then must coincide with points $\mathbf{x}^{\mathrm{E}}$ satisfying $\nabla \mathrm{V}\left(\mathbf{x}^{\mathrm{E}}, \mathbf{x}^{0}\right)=\mathbf{0}$. The operating point of interest, $\mathbf{x}^{0}$, is typically identified before conducting stability studies. Therefore, the dependence of $\mathrm{V}$ on its second argument will be suppressed in the following discussion; we will write $\mathrm{V}(\mathbf{x})$ to replace $\mathrm{V}\left(\mathbf{x}, \mathbf{x}^{0}\right)$. 
Below we outline an iterative algorithm that expands contours described by $\{\mathbf{x} \mid \mathrm{V}(\mathbf{x})=C\}$, seeking to identify the values of $C$ associated with contours intersecting points $x^{E}$ for which the objective function $\left\|\nabla V\left(x^{E}\right)\right\|^{2}$ is equal to 0 . The update at each iteration will use a simple one dimensional search technique to estimate a smallest increment in $\mathrm{C}$ that will tend to drive the objective to zero. An obvious question in such an approach is how to handle multiple local minima of the objective function on the constraint surface. For the objective function chosen above, we will show that in a neighborhood of $\mathbf{x}^{0}$ (i.e., for sufficiently small values of $\mathrm{C}$ ), there must be $2 \mathrm{n}$ local minima on the constraint surface. The proposed approach is to use these $2 \mathrm{n}$ candidate starting points, and attempt to trace out one dimensional paths of local minima as the value of $\mathrm{C}$ is increased.

\section{OUTLINE OF ALGORITHM}

Notation: Iterate numbers will be indicated in square brackets. Multiple instances of a given vector will be indicated by a superscript. For example, $\mathbf{x}^{2}$ [5] would represent the second $\mathbf{x}$ vector, at its fifth iteration. Recall that $\mathbf{x}^{0}$ denotes the stable operating point of interest, and does not change.

0) Starting data:

$C[0]=\varepsilon>0$, a small real constant.

"Initial guesses" for iterative optimization:

$\left\{\mathbf{x}^{1}[0], \mathbf{x}^{2}[0], \ldots, \mathbf{x}^{2 \mathrm{n}}[0]\right\}$.

Each satisfies $V\left(\mathbf{x}^{\mathrm{k}}[0]\right)=\mathrm{C}[0]$.

$\delta$, tolerance threshold; classifying objective $\approx 0$ when

lobjective $\mid<\delta$.

Initialize iteration index at $\mathrm{i}=0$.

1) Identify set $\mathrm{L}$ of indices $\mathrm{k}$ for which $\left\|\nabla \mathrm{V}\left(\mathrm{x}^{\mathrm{k}}[\mathrm{i}]\right)\right\|^{2} \geq \delta$. Save those $\mathbf{x}^{\mathrm{k}}[\mathrm{i}]$ for which $\left\|\nabla \mathrm{V}\left(\mathrm{x}^{\mathrm{k}}[\mathrm{i}]\right)\right\|^{2}<\delta$; these are equilibria. If $\mathrm{L}$ is the empty set, QUIT. Otherwise, identify $\mathrm{k}^{*} \in \mathrm{L}$ for which $\left\|\nabla \mathrm{V}\left(\mathbf{x}^{\mathrm{k}}[\mathrm{i}]\right)\right\|^{2}$ is minimized.

2) Use outward normal of constant Lyapunov surface at $\mathbf{x}^{\mathrm{k}^{*}}[\mathrm{i}]$, $\nabla \mathrm{V}\left(\mathrm{x}^{\mathrm{k} *}[\mathrm{i}]\right)$, as a "search direction." Attempt to drive objective to zero in this direction; i.e., solve for scalar parameter $\gamma$ to minimize:

$$
\left.\| \nabla V\left(\mathbf{x}^{\mathrm{k}^{*}[\mathrm{i}]}+\gamma \nabla V\left(\mathrm{x}^{\mathrm{k}^{*}[\mathrm{i}]}\right)\right)\right) \|^{2}
$$

3) Update $\mathrm{C}^{\mathrm{i}+1}=\mathrm{V}\left(\mathbf{x}^{\mathrm{k}^{*}}[\mathrm{i}]+\gamma \nabla \mathrm{V}\left(\mathrm{x}^{\left.\left.\mathrm{k}^{*}[\mathrm{i}]\right)\right)}\right.\right.$

4) For each $k \in L$, numerically solve the following constrained optimization problem, using $x^{\mathrm{k}}[i]$ as the initial guess.

$$
\text { minimize }\|\nabla V(\mathbf{x})\|^{2}
$$

subject to

$\mathrm{V}(\mathbf{x})=\mathrm{C}[\mathrm{i}+1]$

For each $k \in L$, set $x^{k}[i+1]$ equal to the resulting minimizer.

5) Let $i=i+1$ and return to (1)

\section{INITIALIZING ITERATIVE OPTIMIZATION SCHEME}

A key point in the algorithm outlined above is the selection of the initial values $\left\{\mathbf{x}^{1}[0], \mathbf{x}^{2}[0], \ldots, \mathrm{x}^{2 \mathrm{n}}[0]\right\}$ that serve as starting points for the iterative optimization. As discussed above, it is expected that each of these will provide a first point on a path passing through local minima of $\|\nabla V(\mathbf{x})\|^{2}$ on the successive constant contour surfaces obtained as $\mathrm{C}[\mathrm{i}]$ is increased. A necessary condition for a local minimum in a constrained optimization problem is that the gradient of the objective must be orthogonal to the tangent space of the constraint manifold. The objective and constraint functions here are assumed continuously differentiable; moreover, we will assume that the C[i]'s of interest are regular values for $\mathrm{V}(\mathbf{x})$, so the sets $\{\mathbf{x} \mid \mathrm{V}(\mathbf{x})=C[i]\}$ yield well defined manifolds [8]. The close relationship of the chosen objective function and constraint yields a simple interpretation of this orthogonality condition.

\section{Proposition}

Consider $\hat{\mathbf{x}} \in\{\mathbf{x} \mid \mathrm{V}(\mathbf{x})=\mathrm{C}\}$. If $\hat{\mathbf{x}}$ is a local minimum of $\|\nabla V(\mathbf{x})\|^{2}$ on this manifold, then $\nabla V(\hat{\mathbf{x}})$ is an eigenvector of $\mathrm{J}(\hat{\mathbf{x}}):=\nabla^{2} \mathrm{~V}(\hat{\mathbf{x}})$.

\section{Proof}

The gradient of the objective function is given by:

$$
2 J(\hat{\mathbf{x}}) \nabla V(\hat{\mathbf{x}}) \text {. }
$$

The outward normal of the constraint manifold is simply:

$$
\nabla V(\hat{\mathbf{x}})
$$

If $\hat{\mathbf{x}}$ is a local minimum, then at this point the gradient of the objective function must be co-linear with the outward normal of the constraint manifold. It follows that there exists a real scalar constant $\lambda$ such that

$$
\mathrm{J}(\hat{\mathbf{x}}) \nabla \mathrm{V}(\hat{\mathbf{x}})=\lambda \nabla \mathrm{V}(\hat{\mathbf{x}}) .
$$

To use this result to identify candidate local minima, we must assume that $\mathrm{C}[0]$ is chosen "sufficiently small;" i.e., the size of $\mathrm{C}[0]$ must be such that on the set $\{\mathbf{x} \mid \mathrm{V}(\mathbf{x})=\mathrm{C}[0]\}, \nabla V\left(\mathbf{x}^{0}+\Delta \mathbf{x}\right)$ is well approximated by its linearization $J\left(x^{0}\right) \Delta \mathbf{x}$. Under these conditions, consider a point $\hat{\mathbf{x}}$ obtained at one of the two intersections between the manifold $\{\mathbf{x} \mid \mathrm{V}(\mathbf{x})=\mathrm{C}[0]\}$ and the \pm directions given by an eigenvector $\mathbf{v}$ of $\mathrm{J}\left(\mathbf{x}^{0}\right)$ (note: this eigenvector is translated to pass through $\mathbf{x}^{0}$ ). Let $\lambda$ denote the corresponding eigenvalue, and $\Delta \mathbf{x}$ denote $\hat{x}-x^{0}$. Note that $\Delta \mathbf{x}$ will be co-linear with $\mathbf{v}$. Then observe that $\nabla V(\hat{\mathbf{x}}) \approx \mathbf{J}\left(\mathbf{x}^{0}\right) \Delta \mathbf{x}=$ $\lambda \Delta \mathbf{x}$; up to the accuracy of the linear approximations, $\nabla \mathrm{V}(\hat{\mathbf{x}})$ will be co-linear with an eigenvector of $\mathrm{J}(\hat{\mathbf{x}})$. This suggests that the $2 \mathrm{n}$ points obtained by intersecting teigenvectors of $J\left(\mathbf{x}^{0}\right)$ with $\{\mathbf{x} \mid V(\mathbf{x})=C[0]\}$ should serve as initial guesses $\left\{\mathbf{x}^{1}[0], \mathbf{x}^{2}[0], \ldots\right.$, $\left.\mathrm{x}^{2 \mathrm{n}}[0]\right\}$ for the optimization algorithm described above. Straightforward differentiation of the objective function will reveal that its Hessian is positive definite in a neighborhood of $\mathbf{x}^{0}$, so all of these points will be (approximate) local minima. A conceptual sketch of the role of these eigenvector directions, the constraint surface, and the objective function is shown in Figure 1. 


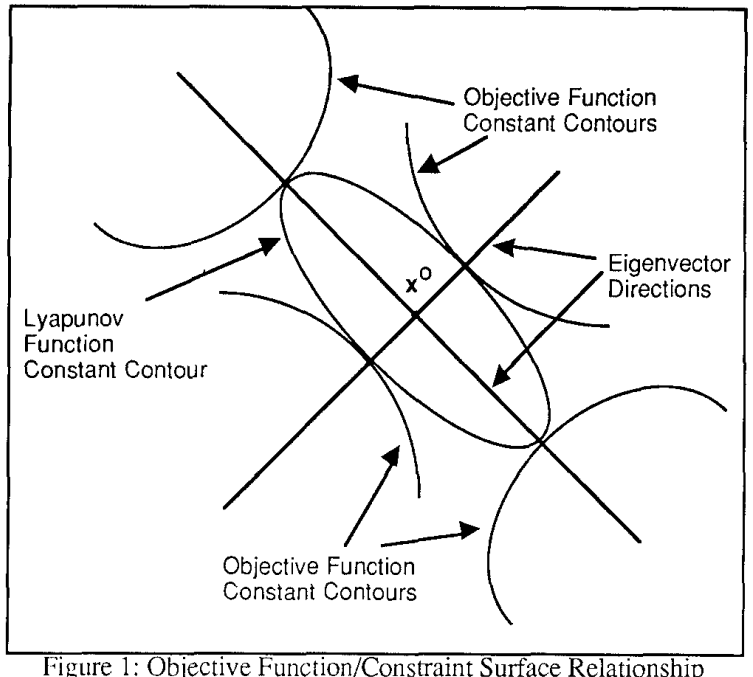

Figure 1: Objective Function/Constraint Surface Relationship
[4] C. L. DeMarco and A. R. Bergen, "On Locating Unstable Equilibrium Points for Power System Transient Stability Analysis," U. S. Department of Energy Report DOE/ET/29364-T1, 1982.

[5] H-D. Chiang and J. S. Thorp, "The Closest Unstable Equilibrium Point Method for Power System Dynamic Security Assessment," IEEE Trans. Circuits and Systems, v. 36, no. 9, pp. 1187-1200, Sept. 1989.

[6] C. L. DeMarco, "A New Method of Constructing Lyapunov Functions for Power Systems," Proc. 1988 IEEE Int. Symp. Circuits and Systems, pp. 905-908, Helsinki, Finland, June 1988.

[7] C. L. DeMarco and T. J. Overbye, "An Energy Based Security Measure for assessing Vulnerability to Voltage Collapse," to appear, IEEE Trans. on Power Systems., also, IEEE Power Engineering Society, Paper 89 SM 712-1 PWRS, July 1989.

[8] V. Guillemin and A. Pollack, Differential Topology, Prentice-Hall, Englewood Cliffs, N. J., 1974.

\section{CONCLUSIONS}

This paper has proposed an approach to locating unstable equilibrium points in a class of power system models, ranking these equilibria in terms of a scalar Lyapunov function. The approach draws heavily on the geometric insight that constant contours of the system Lyapunov function form nested sets, and the algorithm seeks to move outward on these contours, using a constrained optimization on each surface to locate points were the norm squared of the Lyapunov function gradient is minimized. A key result is to bound the number of local minima that may exist. The paper shows that in a neighborhood of the operating point, there will exist $2 n$ local minima, where $n$ is the dimension of the state. This local result yields $2 n$ starting points for the proposed algorithm. The proposed iteration can then be viewed as a homotopy method that attempts to trace out one dimension paths of local minima of the norm squared objective function. Future work will examine numerical implementation of this approach.

\section{ACKNOWLEDGEMENTS}

The support of this work by the Electric Power Research Institute Project RP 2675-4 and National Science Foundation grant ECS-8857019 is gratefully acknowledged.

\section{REFERENCES}

[1] A. H. El-Abiad and K. Nagappan, "Transient stability regions of multimachine power systems," IEEE Trans. Power App. and Sys., v. PAS-85, pp. 169-179, Feb. 1966.

[2] G. E. Gless, "Direct Method of Lyapunov Applied to Transient Power System Stability," IEEE Trans. Power App. and Sys., v. PAS-85, pp. 159-168, Feb. 1966.

[3] G. A. Lüders, "Transient Stability of Multimachine Power Systems via the Direct Method of Lyapunov," IEEE Trans. Power App. and Sys., v. PAS-90, no. 1, pp. 23-36, Jan. 1971. 